\title{
CPAP de Boussignac en procedimientos diagnóstico-terapéuticos en pacientes críticos
}

\author{
M.L. Gómez Grande ${ }^{\mathrm{a}, *}$ y J. Lázarob \\ a Unidad de Cuidados Intensivos, Hospital General Universitario de Ciudad Real, Ciudad Real, España \\ b Servicio de Neumología, Hospital General Universitario de Ciudad Real, Ciudad Real, España
}

Recibido el 4 de abril de 2010; aceptado el 16 de julio de 2010

\author{
PALABRAS CLAVE \\ CPAP Boussignac; \\ Fibrobroncoscopia; \\ Endoscopia; \\ Hemorragia digestiva; \\ Insuficiencia \\ respiratoria aguda; \\ Edema agudo de \\ pulmon
}

\section{KEYWORDS}

Boussignac CPAP;

Bronchoscopy;

Endoscopy;

Systemic;

Digestive

hemorrhage;

Acute respiratory

failure;

Acute pulmonary

edema

\begin{abstract}
Resumen La válvula de Boussignac se ha convertido en un elemento a tener en cuenta en el manejo el edema agudo de pulmón. Gracias a su capacidad para generar presión positiva continua en la vía aérea (CPAP) y su simplicidad, su utilización se está extendiendo en los servicios de emergencias y de urgencias y las plantas de hospitalización. Consiste en un pequeño tubo que se coloca sobre el puerto espiratorio de la mascarilla. El flujo (oxígeno/aire) se acelera al pasar a través de cuatro microcanales que existen en la pared de la válvula, y por el principio de la energía en movimiento, consiguen presurizar el árbol bronquial. Su sistema de funcionamiento, no hermético, permite el paso de una sonda a través de ella para asistir al paciente (beber, alimentarse, aspirar, etc.). Ello la capacita para ampliar su utilización en pacientes que, estando en situación límite, necesitan pruebas diagnósticas que pudieran empeorar la situación respiratoria, como los procedimientos endoscópicos. Presentamos a 3 pacientes críticos a los que se administró CPAP con una válvula de Boussignac acoplada a una mascarilla orofacial durante la realización de dos fibrobroncoscopias y una endoscopia digestiva alta. Los 3 casos culminaron con éxito y no se necesitó la intubación orotraqueal.

๑ 2010 Elsevier España, S.L. y SEMICYUC. Todos los derechos reservados.
\end{abstract}

Boussignac CPAP in diagnostic-therapeutic procedures in the critical patient

Abstract Boussignac valve is a new resource to consider in acute pulmonary edema cardiogenic management. As it generates positive airways pressure continuous (CPAP) and it is very simple to use, its use is being extended to emergency, urgency and hospitalization ward services. This valve is a small tube placed over the interface expiratory port. The gas flow (oxygen/air) accelerates when it crosses through four microchannels in the valve wall, pressurizing the bronchial tree by the principle of the energy in movement. It has a functioning, not hermetic system, allowing the passage of a catheter through it to help the patient (to drink, to eat, to aspirate, etc.). These characteristics make it possible to extend it use in those patients who, in extreme conditions, need diagnostic tests that could deteriorate their respiratory situation,

\footnotetext{
* Autor para correspondencia.

Correo electrónico: mlgomezgrande2000@yahoo.es (M.L. Gómez Grande).
} 
such as endoscopy procedures. We report three critical patients who received CPAP with a Boussignac valve connected to an orofacial interface, while performing two fibrobronchoscopies and one upper digestive tract endoscopy. All finished successfully and none of them need orotracheal intubation.

(c) 2010 Elsevier España, S.L. and SEMICYUC. All rights reserved.

\section{Introducción}

Varios metaanálisis han demostrado el efecto favorable de la presión positiva continua en la vía aérea (CPAP) en la reducción de la mortalidad y la necesidad de intubación orotraqueal (IOT) en pacientes con edema agudo de pulmón (EAP). Sin embargo, en 2008 se publicó un trabajo que incluyó a más de 1.000 pacientes ${ }^{1}$ y observó una mejoría en los parámetros metabólicos, pero no de la mortalidad. Más recientemente, un metaanálisis ${ }^{2}$ ha concluido que la evidencia aún confirma una disminución de la mortalidad y la necesidad de IOT en pacientes aquejados de EAP que reciben soporte respiratorio con CPAP, especialmente cuando su origen es isquémico. La utilización de la CPAP de Boussignac (CPAP-B) se está extendiendo a diferentes niveles de la asistencia sanitaria para atender el EAP, especialmente en el ambiente extrahospitalario, urgencias y salas de hospitalización, al ser un dispositivo no mecánico, de fácil aplicación y transporte, que puede administrar presiones suficientes para manejar al paciente.

La realización de procedimientos endoscópicos con fines diagnóstico-terapéuticos en pacientes con hipoxia grave en respiración espontánea suele precipitar un empeoramiento de la hipoxia (caída de la presión parcial de oxigeno $\left[\mathrm{pO}_{2}\right]$ entre $10-20 \mathrm{mmHg})^{3,4}$ que puede conducir a la IOT y conexión a ventilación mecánica, e incluso pueden aparecer arritmias e inestabilidad hemodinámica ${ }^{5,6}$. La literatura ha descrito la realización de la fibrobroncoscopia con fines diagnósticos asistida por ventilación no invasiva (VNI), en un intento de reclutar alvéolos y mejorar la ventilación, con buenos resultados $^{7}$. Asimismo se ha publicado un estudio aleatorizado en pacientes con $\mathrm{pO}_{2} / \mathrm{FiO}_{2}<300$ a los que se realizaba una fibrobroncoscopia diagnóstica asistida con mascarilla orofacial y válvula de Boussignac como método para conseguir CPAP ${ }^{8}$.

\section{Métodos}

Presentamos a 3 pacientes ingresados en una unidad de cuidados intensivos $(\mathrm{UCl})$ con insuficiencia respiratoria aguda hipoxémica en los que se necesitó realizar una técnica endoscópica, bien con fines diagnósticos, bien con fines terapéuticos. Todos los pacientes estaban ingresados en una $\mathrm{UCI}$ y recibían oxígeno con mascarilla Venturi al 50\% (10 lpm) o mascarilla reservorio con 15 lpm; todos estaban monitorizados estrechamente (ECG, FR, pulsioximetría, presión arterial invasiva, excepto el caso 3 , que fue no invasiva). Una vez indicado el procedimiento, se aplicó el siguiente protocolo: $30 \mathrm{~min}$ antes de iniciar el procedimiento, se colocó la CPAP-B ajustando el flujo de oxígeno para conseguir una presión de $10 \mathrm{cmH}_{2} \mathrm{O}$, monitorizada durante todo el procedimiento para evitar variaciones derivadas de la introducción de la sonda endoscópica. En el momento de iniciar el procedimiento, se administró lidocaína tópica en fosas nasales, vía aérea superior y árbol bronquial. Nuevamente se colocó la mascarilla y se administró midazolam $0,1 \mathrm{mg} / \mathrm{kg}+0,5 \mathrm{mg}$ de fentanilo intravenosos para conseguir sedoanalgesia. Se introdujo el fibrobroncoscopio (Olympus Evis Exera BF-160) y, tras su retirada, se dejó la CPAP 30 min más. En el caso de la endoscopia digestiva, se introdujo el endoscopio (Olympus Eux Exera EU-M60) en la cavidad bucal, tras administrar adrenalina tópica en las fauces.

\section{Caso 1}

Mujer de 73 años que había ingresado en la planta de hospitalización de medicina interna tras haber sido intervenida por una diverticulitis aguda perforada con absceso perisigmoideo. La causa de ingreso en $\mathrm{UCl}$ fueron crisis tonicoclónicas de repetición, por lo que necesitó IOT. Posteriormente, se observó que la causa de las crisis era un bajo gasto cardiaco secundario a un trastorno del ritmo cardiaco (bloqueo auriculoventricular intrahisiano I grado III paroxístico), por lo que se implantó un marcapasos DDDR. Al sexto día de la intubación pudo extubarse, y en las primeras horas postextubación presentó una atelectasia masiva izquierda, con un cociente $\mathrm{pO}_{2} / \mathrm{FiO}_{2}$ de 120 con mascarilla Venturi $50 \%$ (10 lpm), por lo que se realizó una fibrobroncoscopia con CPAP-B. Se aplicó el protocolo descrito y se pudo realizar el procedimiento con éxito; se aspiró gran cantidad de secreciones y se resolvió la atelectasia. Se realizaron gasometría (tabla 1) y radiografía de tórax (fig. 1) antes y después del procedimiento. En los cultivos posteriores de las muestras obtenidas, se aisló Acinetobacter baumanni, y se consideró colonización, dada la ausencia de otros signos de infección (fiebre, leucocitosis, nuevos infiltrados en la radiografía de tórax).

\section{Caso 2}

Mujer de 29 años diagnosticada de lupus eritematoso sistémico que había ingresado en la planta de hospitalización por insuficiencia respiratoria y anemización (hemoglobina, $5,3 \mathrm{gr} / \mathrm{dl}$ ). Unas horas después ingresó en UCI por un nuevo episodio de sangrado alveolar con inestabilidad respiratoria y un cociente $\mathrm{pO}_{2} / \mathrm{FiO}_{2}$ de 140 (Ventimask 50\% 10 (pm). A las $24 \mathrm{~h}$ del ingreso, se realizó fibrobroncoscopia diagnóstica, con lavado broncoalveolar (LBA) utilizando una CPAP-B según el protocolo descrito, sin complicaciones y obteniendo un contenido hemático cuyo cultivo fue negativo. Los datos de las muestras de gasometría antes y después de la fibrobroncoscopia se muestran en la tabla 1. 

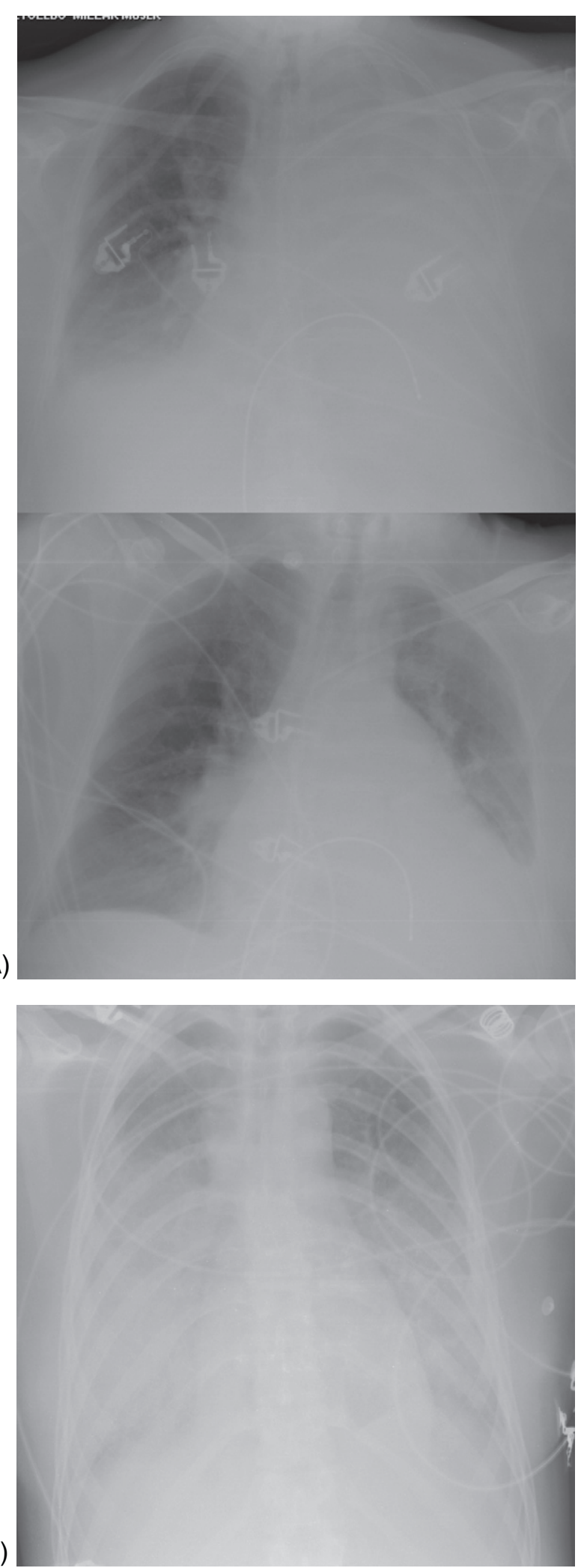

(B)

Figura 1 A: radiografía de tórax antes y después de la fibrobroncoscopia en el paciente 1. B: radiografía de tórax en el paciente 2 previa a la fibrobroncoscopia.

\section{Caso 3}

Varón de 57 años, sin antecedentes de interés, que acudió al servicio de urgencias hospitalario por dolor torácico. Se diagnosticó un síndrome coronario agudo con ascenso del segmento ST en la cara anterior cardiaca y se realizó una coronariografía percutánea. El paciente presentaba una lesión en la porción media de la arteria coronaria descendente anterior, por lo que se realizó angioplastia y se colocó un stent, sin complicaciones, tras haber administrado $300 \mathrm{mg}$ de ácido acetilsalicílico, $300 \mathrm{mg}$ de clopidogrel y $5.000 \mathrm{Ul}$ de heparina sódica intravenosa. Al final del procedimiento el paciente estaba en Killip III y presentó una hematemesis masiva. La saturación arterial de $\mathrm{O}_{2}$ era del 92\%, con un Ventimask al 50\% $10 \mathrm{lpm}$. Se realizó una endoscopia digestiva con soporte respiratorio con CPAP-B, según el protocolo descrito, y se observó una lesión de Mallory Weiss. La hemoglobina cayó de 15,1 a $13 \mathrm{~g} / \mathrm{dl}$ y el hematocrito del 43,6 al 39,1\%. Al finalizar el procedimiento, el paciente presentaba saturación de $100 \%$ y pudo retirarse la CPAP-B (no se realizó gasometría arterial por el riesgo de sangrado tras la administración de anticoagulantes y antiagregantes).

\section{Discusión}

En ocasiones, los pacientes críticos necesitan pruebas diagnósticas que pueden empeorar su estado. En estos casos hay que elegir entre el riesgo y la rentabilidad de la prueba. En los pacientes críticos no intubados, la hipoxemia grave contraindica la realización de una broncoscopia, ya que la $\mathrm{pO}_{2}$ disminuye habitualmente de 10 a $20 \mathrm{mmHg}$ después de una broncoscopia no complicada ${ }^{3,4}$, lo que favorece la aparición de insuficiencia respiratoria grave o arritmias cardiacas ${ }^{5,6}$. Concretamente, la American Thoracic Society ${ }^{9}$ contraindica la realización de la fibrobroncoscopia en pacientes no intubados con hipoxia grave y respiración espontánea $\left(\mathrm{FiO}_{2}\right.$ 0,5 para mantener al menos una $\mathrm{pO}_{2}$ de $75 \mathrm{mmHg}$ ). En los pacientes críticos en situación respiratoria límite, existen tres opciones: intubar al paciente, no realizar la prueba o realizarla en ventilación no invasiva. En 1996 Antonelli et $\mathrm{al}^{8}$ evaluaron la factibilidad y la seguridad de realizar fibrobroncoscopia y LBA en 8 pacientes inmunodeprimidos con afección importante del intercambio gaseoso utilizando ventilación no invasiva como soporte respiratorio (respirador servo 900C) sin grupo control. Para ello seleccionaron a los pacientes inmunodeprimidos con sospecha de neumonía e hipoxemia grave $\left(\mathrm{pO}_{2} / \mathrm{FiO}_{2} \leq 100\right)$. Utilizaron la combinación de CPAP $\left(4 \mathrm{cmH}_{2} \mathrm{O}\right)$ para reclutar alvéolos y presión de soporte para aumentar la ventilación y aumentar la presión de la vía aérea media; utilizaron $\mathrm{FiO}_{2}{ }^{1}$. Ningún paciente necesitó intubación orotraqueal o reinicio de la VNI tras $96 \mathrm{~h}$ del procedimiento. El mismo autor, en $2002^{10}$ publicó un trabajo realizado en 4 pacientes con $\mathrm{pO}_{2} / \mathrm{FiO}_{2}<200$ utilizando como interfase el helmet, en los que se realizó la fibrobroncoscopia con fines diagnósticos, y resultó un método seguro al mantener el intercambio de gases. Ningún paciente necesitó IOT en las siguientes $24 \mathrm{~h}$. Otro trabajo realizado en pacientes con insuficiencia respiratoria aguda hipoxémica $\left(\mathrm{pO}_{2} / \mathrm{FiO}_{2}<200\right)^{11}$ aleatorizó a 26 pacientes a recibir tratamiento estándar o VNI durante la realización de una broncoscopia con fines diagnósticos; se concluyó que la 
Tabla 1 Monitorización clínico-gasométrica de los pacientes

\begin{tabular}{|c|c|c|c|c|c|c|}
\hline & \multicolumn{2}{|c|}{ Caso 1} & \multicolumn{2}{|c|}{ Caso 2} & \multicolumn{2}{|c|}{ Caso 3} \\
\hline & Pre-FB & Post-FB & Pre-FB & Post-FB & Pre-FB & Post-FB \\
\hline FC (lpm) & 118 & 90 & 103 & 95 & 110 & 78 \\
\hline FR (rpm) & 35 & 24 & 38 & 16 & 32 & 16 \\
\hline $\mathrm{SatO}_{2}(\%)$ & 95 & 100 & 89 & 100 & 92 & 100 \\
\hline $\mathrm{PA}(\mathrm{mmHg})$ & $163 / 95$ & $120 / 65$ & $135 / 50$ & $130 / 60$ & $105 / 59$ & $110 / 55$ \\
\hline $\mathrm{FiO}_{2}(\%)$ & 50 & 50 & 50 & 40 & 50 & 24 \\
\hline $\mathrm{pH}$ & 7,44 & 7,41 & 7,33 & 7,43 & & \\
\hline $\mathrm{PCO}_{2}(\mathrm{mmHg})$ & 43 & 42 & 46 & 34 & & \\
\hline $\mathrm{PO}_{2}(\mathrm{mmHg})$ & 62 & 210 & 77 & 187 & & \\
\hline $\mathrm{HCO}_{3}(\mathrm{mmol} / \mathrm{l})$ & 29 & 26 & 24 & 22,6 & & \\
\hline EB & 4,5 & 1,7 & $-1,9$ & $-1,2$ & & \\
\hline $\mathrm{SatO}_{2}(\%)$ & 95 & 100 & 91 & 100 & & \\
\hline Ácido láctico (mg/dl) & 10 & 13 & 17 & 14 & & \\
\hline
\end{tabular}

EB: exceso de base; FC: frecuencia cardiaca; FR: frecuencia respiratoria; Ipm: latidos por minuto; rpm: respiraciones por minuto; SatO ${ }_{2}$ : saturación arterial de oxígeno.

realización de una broncoscopia en pacientes con hipoxia grave en ventilación no invasiva fue superior en cuanto a intercambio de gases y tolerancia hemodinámica que cuando se realizó con tratamiento estándar con suplementos de oxígeno.

La válvula de Boussignac (Vygon, Ecouen, Francia) ${ }^{12}$ consiste en un dispositivo que contiene en su pared capilares que aceleran el flujo administrado (fig. 2). Su funcionamiento se basa en el principio de la conservación de la energía del movimiento. La aceleración del gas en los capilares y la velocidad de los jets se obtienen de una fuente de flujo, una mezcla de gas o aire- $\mathrm{O}_{2}$ del exterior, con una presión de 3,5 bar, y se puede conseguir una CPAP de 2,5$10 \mathrm{cmH}_{2} \mathrm{O}$. El peak-flow de la CPAP está entre 0 y $300 \mathrm{l} / \mathrm{min}$. La cantidad de gas que entra en el circuito regula la presión de la CPAP y será directamente proporcional a la cantidad de gases administrados por el médico. La tabla 2 muestra algunas de las características de la CPAP-B.

Diversas publicaciones han comunicado la utilidad de la CPAP-B en el edema agudo de pulmón ${ }^{13-15}$ y su aplicación

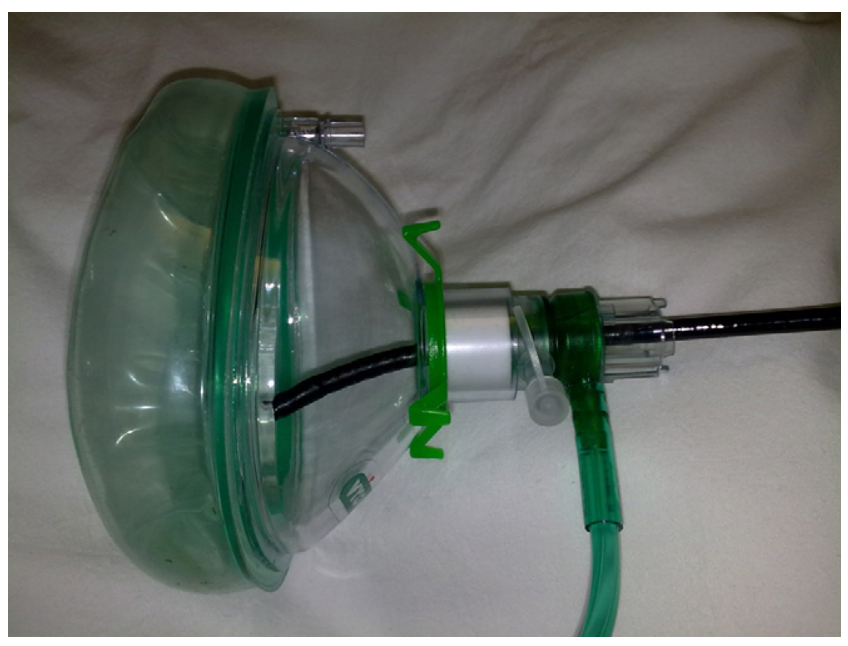

Figura 2 Válvula de Boussignac conectada a una mascarilla. Fibrobroncoscopio a través de la válvula. en los servicios de emergencias ${ }^{16,17}$. Sin embargo, su utilidad podría extenderse a pacientes críticos en los que la realización de una prueba diagnóstica (fibrobroncoscopias, endoscopia digestiva o ecocardiograma trasesofágico) puede deteriorar su situación respiratoria hasta necesitar IOT.

En 2000, Maitre et $\mathrm{al}^{7}$ realizaron un estudio prospectivo y aleatorizado para comparar la realización de fibrobroncoscopia con fines diagnósticos y utilizaron una CPAP-B en pacientes con $\mathrm{pO}_{2} / \mathrm{FiO}_{2}<300 \mathrm{mmHg}$. En el grupo control, el flujo de oxígeno se administraba por el puerto accesorio (presión 0) que habitualmente se utiliza para medir la presión generada. En el grupo de tratamiento se administraba en la entrada de la válvula, presurizando el sistema desde 2,5 a 7,5 $\mathrm{cmH}_{2} \mathrm{O}$, y el oxígeno se ajustó hasta la misma concentración que en el otro grupo. Tras finalizar el procedimiento, el sistema se mantuvo $30 \mathrm{~min}$. Se incluyó a 30 pacientes; 5 pacientes del grupo que recibió oxígeno sin CPAP necesitaron intubación en las $6 \mathrm{~h}$ siguientes y ninguno del grupo que recibió CPAP. No hubo diferencias entre los pacientes que recibieron LBA y los que no. Los autores concluyen que el dispositivo puede ser útil para evitar el deterioro de los pacientes con insuficiencia respiratoria durante la realización de la fibrobroncoscopia.

Presentamos 3 casos en los que la realización del procedimiento endoscópico en situación límite $\left(\mathrm{pO}_{2} / \mathrm{FiO}_{2}<150\right)$

Tabla 2 Ventajas de la CPAP de Boussignac
Simplicidad de uso
Suministro de altas concentraciones de oxígeno, posibilidad de realizar mezcla de gases
Monitorización de presión
Imposibilidad de reinhalación de anhídrido carbónico
Bajo espacio muerto
Sistema abierto al exterior que permite el paso de una sonda de aspiración, sonda de alimentación, sonda endoscópica, etc.
Sistema desechable
Buena tolerabilidad
Bajo coste 
asistido con CPAP-B resultó exitosa gracias a una cuidadosa monitorización y tratamiento, sin necesidad de llegar a la IOT. Si bien la monitorización de la saturación arterial de $\mathrm{O}_{2}$ fue continua, no lo fue la $\mathrm{pCO}_{2}$, que únicamente se pudo monitorizar antes y después del procedimiento de forma invasiva en los casos 1 y 2 .

La CPAP administrada con válvula de Boussignac puede ser una alternativa útil durante la realización de pruebas endoscópicas diagnósticas o terapéuticas en pacientes con insuficiencia respiratoria aguda grave, siempre que lo realice personal experimentado y con una monitorización adecuada; así se puede evitar la IOT y la conexión a ventilación mecánica. Actualmente, se ha comercializado una mascarilla específica con dos puertos, uno para la conexión de la válvula de Boussignac y el otro para el paso de la sonda endoscópica. Al igual que otro tipo de mascarillas, podrían ser utilizadas con dos niveles de presión durante estos procedimientos.

\section{Conflicto de intereses}

Los autores declaran no tener ningún conflicto de intereses.

\section{Bibliografía}

1. Gray A, Goodacre S, Newby DE, Masson M, Sampson F, Nicholl $\mathrm{J}$, for the 3CPO Trialists. Noninvasive Ventilation in Acute Cardiogenic Pulmonary Edema. N Engl J Med. 2008;359: 142-51.

2. Weng $\mathrm{CL}$, Zhao YT, Liu QH, Fu CJ, Sun F, Ma YL, et al. Meta-analysis: noninvasive ventilation in acute cardiogenic pulmonary edema. Ann Intern Med. 2010;152:590-600.

3. Ghows MB, Rosen MJ, Chuang MT, Sacks HS, Teirstein AS. Transcutaneous oxygen monitoring during fiberoptic bronchoscopy. Chest. 1986;89:543-4.

4. Francoz RA, Konopka R, Sgroi V, Moser KM. Changes in ventilation and perfusion in anesthetized dogs following lobar lavage with saline solution. Chest. 1978;74:552-8.

5. Shrader DL, Lakshminarayan S. The effect of fiberoptic bronchoscopy on cardiac rhythm. Chest. 1978;73:821-4.

6. Katz AS, Michelson EL, Stawicki J, Holford FD. Cardiac arrhythmias, frequency during fiberoptic bronchoscopy and correlation with hypoxemia. Arch Intern Med. 1981;141: 603-6.
7. Maitre B, Jaber S, Maggiore SM, Bergot E, Richard JC, Bakthiari $\mathrm{H}$, et al. Continuous positive airway pressure during fiberoptic bronchoscopy in hypoxemic patients. A randomized doubleblind study using a new device. Am J Respir Crit Care Med. 2000;162:1063-7.

8. Antonelli M, Conti G, Riccioni L, Meduri GU. Noninvasive positive-pressure ventilation via face mask during bronchoscopy with bal in high-risk hypoxemic patients. Chest. 1996;110:724-8.

9. American Thoracic Society. Clinical role of bronchoalveolar lavage in adults with pulmonary disease. Am Rev Respir Dis. 1990;142:481-6.

10. Antonelli M, Pennisi MA, Conti G, Bello G, Maggiore SM, Michetti $\mathrm{V}$, et al. Fiberoptic bronchoscopy during noninvasive positive pressure ventilation delivered by helmet. Intensive Care Med. 2003;29:126-9.

11. Antonelli M, Conti G, Rocco M, Arcangeli A, Cavaliere F, Proietti $\mathrm{R}$, et al. Noninvasive positive-pressure ventilation vs conventional oxygen supplementation in hypoxemic patients undergoing diagnostic bronchoscopy. Chest. 2002;121:1149-54.

12. Monchi M. Comparison of the performance of 5 systems of spontaneous ventilation with CPAP on a mechanical lung [tesis doctoral]. Paría: Université Paris VI; 1997.

13. Dieperink W, Iwan CC, Van der Horst A, Jaqueline W, Nannenberg-Koops A, Henk W, et al. A 64-year old man who sustained many episodes of acute cardiogenic pulmonary edema successfully treated with Boussignac continuous positive airway pressure: A case report. Int J Cardiol. 2007;119:268-70.

14. Dieperink W, Nijsten MW, Van der Stadt M, Van der Horst IC, Aarts LP, Zijlstra F, et al. Implementation of Boussignac continuous positive airway pressure in the coronary care unit: experiences and attitudes. Heart Lung. 2008;37:449-54.

15. Dienperik W, Jaarsma T, Van der Horst IC, Nieuwland W, Vermeulen $\mathrm{KM}$, Rosman $\mathrm{H}$, et al. Boussignac continuous positive airway pressure for the managemet of acute cardiogenic pulmonary edema: prospective study with a retrospective control group. BMC Cardiovasc Disord. 2007;7:40.

16. Moritza F, Benichouc J, Vanhestea M, Richardb CH, Linea $S$, Hellotc MF, et al. Boussignac continuous positive airway pressure device in the emergency care of acute cardiogenic pulmonary oedema: a randomized pilot study. Eur J Emerg Med. 2003;10:204-8.

17. Moritz F, Brousse B, Gellée B, Chajara A, L'Her E, Hellot $M F$, et al. Continuous positive airway pressure versus bilevel noninvasive ventilation in acute cardiogenic pulmonary edema: a randomized multicenter trial. Ann Emerg Med. 2007;50:666-75. 